\title{
Cardiac Imaging: Multimodality Advances and Surveillance Strategies in Detection of Cardiotoxicity
}

\author{
Marie Moonen ${ }^{1} \cdot$ Cécile Oury $^{1} \cdot$ Patrizio Lancellotti $^{1,2}$
}

(C) Springer Science+Business Media, LLC 2017

\begin{abstract}
Contemporary cancer management has increased the overall number of cancer survivors, but cardiotoxicity remains a subject of concern, which is a major cause of noncancer mortality among survivors. Among the potential cardiovascular complications, left ventricular (LV) systolic dysfunction is a poor prognostic factor. The importance of its early detection is based on the principle that the likelihood of response to heart failure (HF) treatment is temporally related to the initiation of HF treatment. For these reasons, cardiac monitoring is commonly applied in general practice, based on serial measurements of LV ejection fraction (LVEF); transthoracic echocardiography (TTE) is generally used. However, the LVEF, as a diagnostic and predictive parameter, has significant limitations, which calls for more effective multimodality imaging strategies. This approach requires further study, but there is increasing available data in the literature, encouraging the combination of multimodality imaging parameters and techniques for early cancer therapeutic-related cardiac dysfunction (CTRCD) detection.
\end{abstract}

Keywords Cancer $\cdot$ Cardiotoxicity $\cdot$ Imaging $\cdot$ Biomarkers

This article is part of the Topical Collection on Cardio-oncology

Patrizio Lancellotti

plancellotti@chu.ulg.ac.be

1 GIGA Cardiovascular Sciences, Departments of Cardiology, Cardio-Oncology Clinic, CHU Sart Tilman, University of Liège Hospital, 4000 Liège, Belgium

2 Gruppo Villa Maria Care and Research, Anthea Hospital, Bari, Italy

\section{Introduction-Scope of the Problem}

The multidisciplinary specialty of cardio-oncology is focused on the cardiovascular care of patients with cancer either before or during the course of therapy, or after the completion of treatment [1•]. The two disciplines of cardiovascular and oncologic disease share common points; both are the leading causes of death in the Western world and have some potentially overlapping risk factors. The development of effective screening, alongside advances in cancer therapy, has resulted in a 20\% decline of cancer mortality [2]. However, the price to be paid for such success is the increasing potential for anticancer therapy toxicity. Cardiotoxicity can encompass a broad range of effects: myocardial injury leading to symptomatic or asymptomatic heart failure (HF) with preserved or reduced left ventricular ejection fraction (LVEF), arrhythmias, myocardial ischemia, hypertension, venous thrombotic complications, and metabolic complications. These sequelae can potentially transform cancer treatment into a chronic cardiovascular disease [3]. At present, cardiotoxicity has been associated with anthracyclines, monoclonal antibodies, tyrosine kinase inhibitors, proteasome inhibitors, anti-angiogenesis agents, and immunotherapy agents [4]. A classification system has been proposed to distinguish drugs that have the potential to induced irreversible cardiac damage (type I) versus drugs that predominantly induce reversible dysfunction (type II) [5]. The agents classically associated with type I cardiotoxicity include anthracyclines, whereas the characteristic agent for type II toxicity is trastuzumab. Anthracycline-induced HF conveys a very poor prognosis; compared with idiopathic cardiomyopathy, the survival of patients with cardiomyopathy due to doxorubicin is significantly worse (hazard ratio, 2.64; $95 \%$ confidence interval, 1.35 to $5.17 ; P=0.005$ ) [6]. This high mortality can be partially attributed to noncardiac causes, but distinct outcomes related to advanced therapies for end-stage 
$\mathrm{HF}$ in this patient population has been demonstrated due to unique demographic characteristics and comorbidities [7]. Beyond the risk of symptomatic HF, a substantial proportion of patient can experience asymptomatic changes in LVEF. This cancer therapeutic-related cardiac dysfunction (CTRCD) is reported in 2-3\% in randomized trials on breast cancer women treated with anthracyclines and trastuzumab but can reach up to $26 \%$ in observational studies [8]. There is data to suggest the potential irreversibility of LV dysfunction despite the discontinuation of the cardiotoxic agent, and the likelihood of recovery has been linked to early introduction of HF therapy. Therefore, there is growing interest in strategies in detecting early CTRCD [9]. Currently, the generally accepted method to monitor and detect LV systolic dysfunction during the administration of cardiotoxic agents is the serial measurement of LVEF. Historically, multiple gate acquisition (MUGA) scintigraphy have been used for years with excellent accuracy and reproducibility [10], but has become less utilized in favor of echocardiography. Echocardiography is radiation free, which is of importance considering the specific need of serial monitoring, and offers the great advantage of a complete cardiac evaluation, including assessment of hemodynamic function, valvular function, pericardial disease, and cardiac chamber (i.e., atria, ventricles) size and function. In addition, its recent technological progress, with the appearance of real-time three-dimensional (3D) echocardiography and echography-dedicated contrast agents, has made this increasingly the more preferred technique in all patients before and during cancer treatment.

\section{Cardiotoxicity Definition}

Providing a precise definition of cardiotoxicity is critical, since it could influence the interruption or modification of cancer treatment planning. The ideal definition should be based on one or eventually more parameters that can be easily and noninvasively assessed, with low cost and high accuracy and reproducibility.

LVEF has been until now the only used parameters for cancer therapeutic-related cardiac dysfunction definition. The heterogeneity of LVEF cutoff values used for the diagnostic criteria of CTRCD reported in the literature resulted in difficulty comparing and harmonizing findings across studies. In a joint position paper, the American Society of Echocardiography (ASE) and the European Association of Cardiovascular Imaging (EACVI) recommended a homogeneous CTRCD definition as a decrease of the LVEF of $>10$ percentage points, to a value $<53 \%$, confirmed by a repeated study 2 to 3 weeks after the first diagnostic imaging study $[11 \bullet \bullet$. This definition no longer takes into account signs and symptoms of HF, as it has previously been the case.
However, LVEF, as a diagnostic and predictive parameter, has significant limitations. LVEF is affected by loading conditions and is of limited accuracy for detecting subclinical myocardial dysfunction, and can be prone to significant interobserver and intraobserver variability. Indeed, diagnosing a CTRCD based on a decrease of LVEF could be late, with potential irreversible damage to the heart already done; thus, a more precise definition of cardiotoxicity, including criteria for subclinical LV dysfunction, is warranted. It may be necessary to include combination of imaging criteria and circulating biomarkers, employed as a multimodality strategy as an approach throughout the continuum of cancer care-from risk stratification of cardiotoxicity prior to treatment, to detection of early cardiotoxicity during treatment, and for surveillance of late cardiotoxicity in survivors. The different methods of imaging as well as circulating biomarkers will be discussed, along with a review of the literature regarding their utility for the detection of cardiotoxicity.

\section{Imaging Techniques}

\section{Echocardiography}

Transthoracic echocardiography (TTE) is currently the cornerstone in the cardiac imaging evaluation of patients before, during, and after anticancer therapy. It is inexpensive, readily available, reproducible, noninvasive, with no ionizing radiation exposure, and is also safe in patients with renal functional impairment. Furthermore, a complete TTE examination offers, in a single time, information on LV geometry and LV systolic and diastolic functions, together with an evaluation of valvular function and the pericardium. A recommended cardio-oncology echocardiogram protocol has been recently proposed by the ASE/EACVI [11••]. The calculation of LVEF should be performed at each time with the best method available in a given echocardiography lab. According to the above recommendations, the two-dimensional echocardiography (2DE) method of choice for LV volumes quantification and LVEF calculation is the modified biplane Simpson's technique (Fig. 1). Normal LVEF using biplane method of disks is $63 \pm 5 \%$ and LVEF in the range of 53 to $73 \%$ is classified as normal.

However, accurate LVEF quantification can be limited, not only by the conceptual limitations of the LVEF but also by technical considerations; some issues include the nonuniversally applicable geometric assumptions of the 2D LV model, the requirement of good image quality (especially endocardial border delineation), optimal apical views alignment (to identify the true apex), and the load-dependent nature of LVEF assessment, which can be potentially problematic in cancer patients. The specific cardio-oncology requirement of sequential evaluation of LVEF is therefore confronted to 
Fig. 1 The 2DE method of choice for LV volumes quantitation is the biplane method of disks summation (modified Simpson's rule). Volume measurements are derived from tracings of endocardial borders in the apical four-chamber $(\mathbf{a}, \mathbf{b})$ and two-chamber (c, d) views, in enddiastolic (a, c) and end-systolic $(\mathbf{b}, \mathbf{d})$ frames. The LVEF is then calculated: $\mathrm{LVEF}=(\mathrm{LVEDV}-$ LVESV) / LVEDV. LVEDV LV end-diastolic volume, LVESV LV end-systolic volume

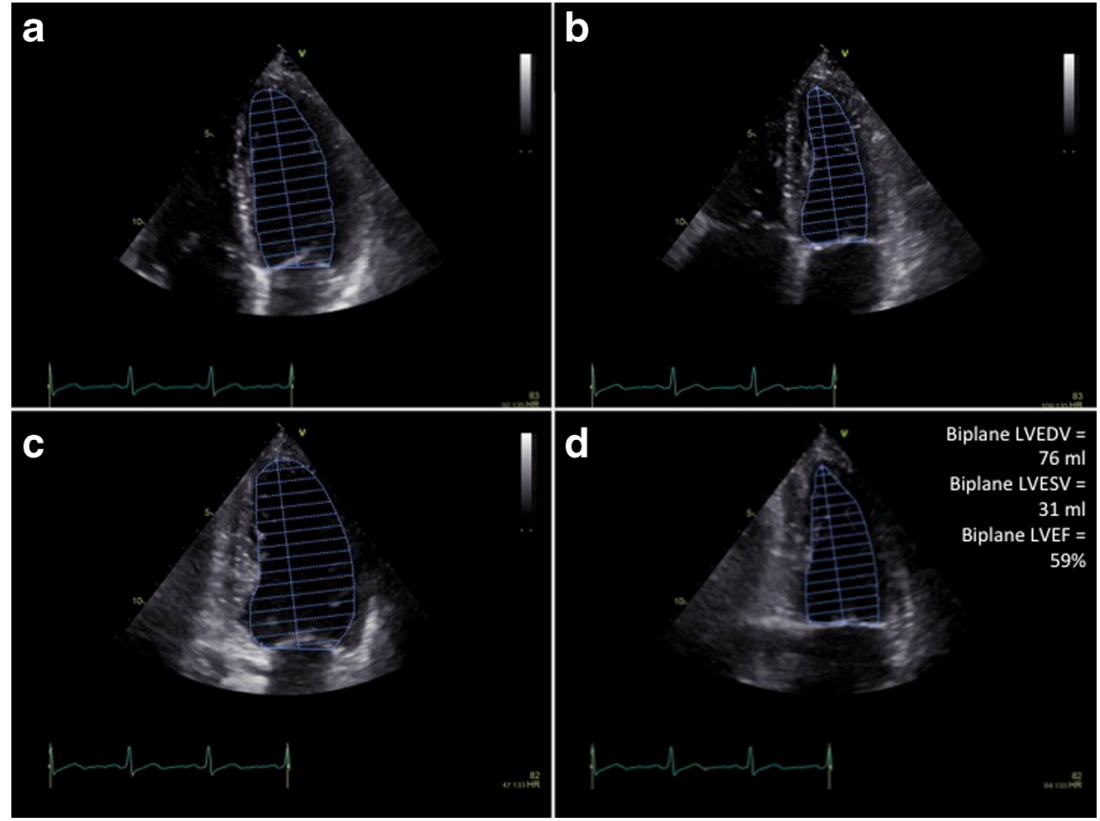

proven significant variability. The sources of variability have been classified as interobserver variability (measurements made on the same images by different investigators), intraobserver variability (repeated measurements made by an individual investigator on the same images), and temporal variability (repeated echo recordings). In a head to head comparison, temporal variability has been shown to represent the dominant component of variation [12]. Indeed, small differences in angulation and placement of the transducer during serial echo recordings may influence measurements of LV dimensions. However, the real life monitoring of LVEF during follow-up of patient faces the interobserver test-retest variability, which is the variability observed between two studies at different time points with measurements performed by different individuals. For 2DE LVEF assessment, the interobserver test-retest variability is very high with minimal detectable difference in EF of 13\% [13], explaining why 2DE LVEF generally fails to detect small variations attributable to cardiotoxicity.

Newer echocardiography techniques, using contrast echocardiography or 3D technology, have resulted in significant improvement in the accuracy of LVEF assessment. Contrast echocardiography is the recommended option when two contiguous LV segments from any apical views are not well seen $[14,15]$. It increases the accuracy of volume assessment in case of poor acoustic window, but it has no demonstrated interest in case of good images quality (lower reproducibility and higher temporal variability) [13]. The potential of 3D echocardiography (3DE) is of upmost interest in the field of cardio-oncology. Indeed, at a time point, 3DE LVEF, compared to 2DE LVEF, has been demonstrated of higher accuracy and of closer correlation to the reference LVEF obtained with cardiac magnetic resonance (CMR) [16, 17]. In an ideal $3 \mathrm{DE}$ assessment of LVEF, the potential errors associated with geometric assumptions, foreshortened views, or suboptimally acquired orthogonal apical two- and four-chamber views are not present, as it is the case with 2DE volume calculations. Furthermore, 3DE LVEF has been demonstrated to have an improved accuracy over 2DE in detecting LVEF $<50 \%$ [18], which is frequently the chosen cutoff value to warrant increased cardiac monitoring during the administration of anticancer agents associated with known cardiotoxicity. 3DE has been showed to be feasible, accurate, and reproducible for assessing changes in LV volumes and LVEF when compared with CMR, in women receiving adjuvant trastuzumab after doxorubicin for breast cancer [19]. The reproducibility of $2 \mathrm{DE}$ and $3 \mathrm{DE}$, with and without contrast agents, has been studied in the breast cancer population, with non-contrast 3DE LVEF demonstrating lower temporal variability [13]. It is recommended that $3 \mathrm{DE}$ LVEF should be the preferred technique for monitoring LV function and detecting CTRCD in patients with cancer [11••] (Fig. 2). However, it is important to consider that 3DE LVEF has the same conceptual limitations as 2D LVEF assessment.

Ultrasound deformation imaging has generated a great deal of interest in detecting subclinical LV dysfunction, which is obtained preferentially by speckle tracking, has been proposed based on its higher sensitivity in detecting subtle systolic LV variation. Also, it is less load dependent than LVEF and may have higher prognostic value. In a multicenter study comparing echocardiographic parameters in patients with HF, global longitudinal strain (GLS) assessment, a parameter evaluating longitudinal aspect of myocardial contraction using deformation imaging, appeared to have a higher and more reliable 
Fig. 2 3D image acquisition focuses primarily on including the entire left ventricle within the pyramidal 3D data set. The software then tracks end-diastolic and end-systolic volume. One of the important advantages of 3D echocardiographic volume measurements is that they do not rely on geometric assumptions. In patients with good image quality, 3D echocardiographic measurements are accurate and reproducible and should therefore be used when available and feasible

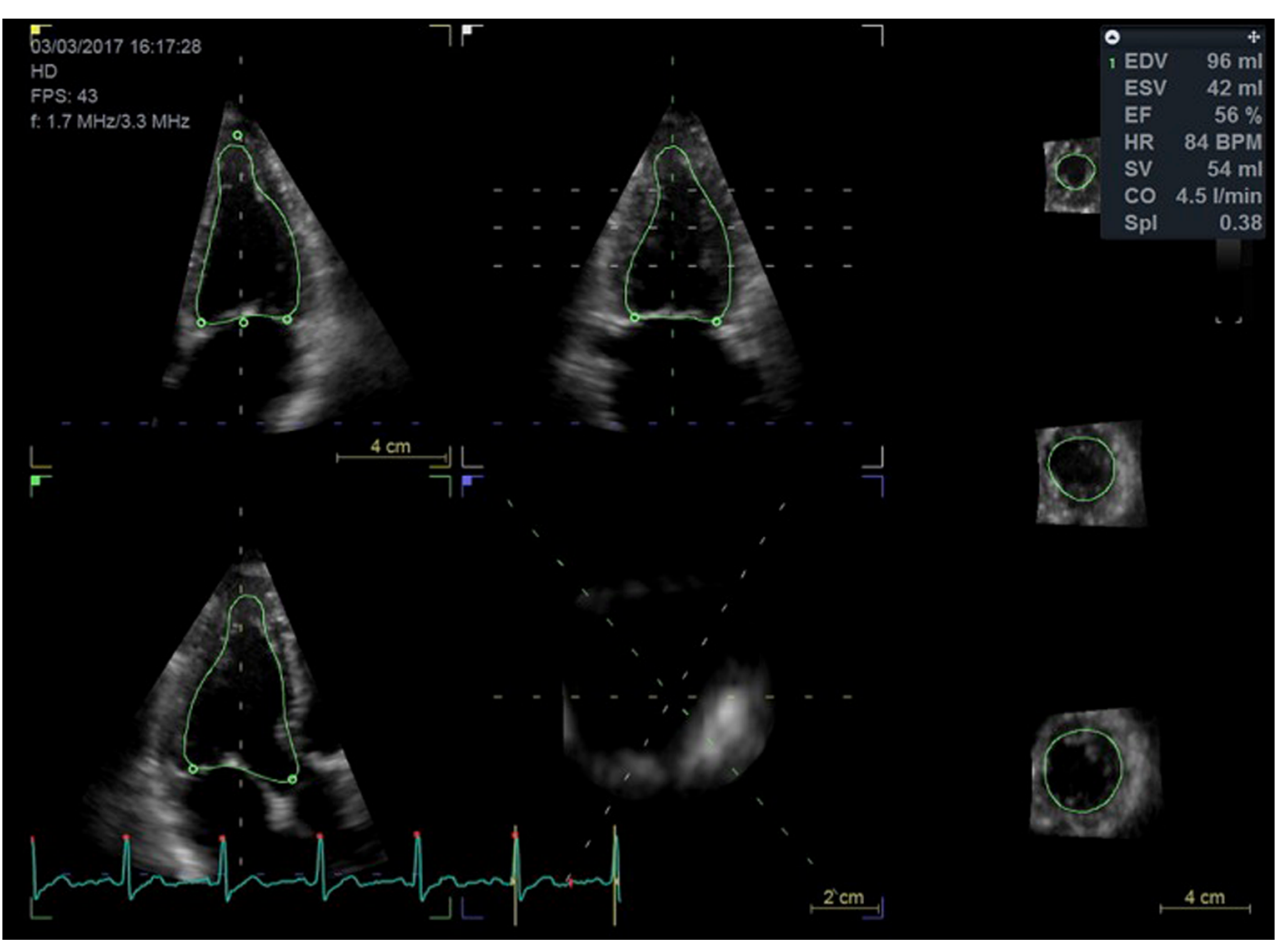

prognostic value than LVEF for the prediction of cardiac events and cardiac deaths [20]. 2D speckle tracking strain has already been evaluated in studies for a total of more than 700 patients concomitantly to the administration of cardiotoxic anticancer agents, with encouraging results concerning its capability to diagnose subclinical LV dysfunction of subsequent clinical relevance [21-27]. Based on these data, serial strain measurements are suggested during anticancer treatment $[11 \bullet \bullet]$. Ideally, the measurements during chemotherapy should always be compared with the baseline value. A relative reduction of GLS of less than $8 \%$ from baseline does not appear to be clinically significant, whereas a decrease of more than $15 \%$ from baseline is likely to be abnormal and predictive of subsequent LVEF decrease and meeting criteria for CTRCD [11••]. If baseline GLS has not been documented, a value of $<-19 \%$ is also predictive of CTRCD. The nadir of the change of GLS has been noted 9 months after the initiation in the case of anthracyclines administration in breast cancer patients treated with anthracycline-trastuzumab regimen [27]. Importantly, the reported GLS should be notified with specification of the used echocardiography machine and software, and with the same vendor-specific echo machine used during follow-up. If the GLS is not measured, then the medial and lateral tissue Doppler systolic velocity (s') and mitral annular plane systolic excursion (MAPSE) should be reported.

\section{Cardiac Magnetic Resonance}

Magnetic resonance imaging (MRI) has gained importance for cardiovascular imaging thanks to advances in both hardware and software. ECG gating and respiratory motion suppression technologies are used to optimize the acquisition of high-quality cross-sectional images of the heart, with spatial resolution of 1-2 $\mathrm{mm}$ and temporal resolution of 25$50 \mathrm{~ms}$. In addition, the application of MRI to the cardiovascular imaging offers a wide field of view and flexible scanning planes. CMR is therefore now considered as the noninvasive reference method for the assessment of cardiac volumes, function, and composition, and for the diagnosis of ischemia and research of myocardial viability. CMR potential is dependent on different techniques, including manipulating of pulse sequence parameters and generating different types of softtissue contrast.

An increasing number of studies have examined CMR capabilities for the evaluation of anticancer therapeutics-induced myocardial remodeling, including LVEF surveillance during anticancer treatment. A CMR technique for the assessment of LV morphology and function is cine steady-state free precession (SSFP), which analyzes a set of contiguous short-axis cine sequences from the atrioventricular plane to the apex, in which the blood pool and myocardium has a bright and dark appearance, respectively. This $3 \mathrm{D}$ volumetric $\mathrm{LV}$ coverage, with high contrast-to-noise ratios, offers images with excellent discrimination of endocardial and epicardial borders, allowing LVEF and mass quantification without the need of geometric assumption, and risk of incomplete sampling of ventricular volumes. Therefore, CMR has been demonstrated to be superior to $2 \mathrm{DE}$ for the quantification of LVEF with higher interstudy reproducibility [28] and has progressively become the gold standard for accurate and reproducible quantification of 
LVEF [29]. Importantly, 2DE and CMR have been compared for the screening of CTRCD in adult survivors of childhood cancer exposed to anthracyclines or left chest radiation [18]. Compared with CMR, 2DE (biplane method) had a sensitivity of $25 \%$ and a false-negative rate of $75 \%$ for detection of LVEF less than $50 \%$. 2DE also overestimated the mean LVEF of this population by up to $5 \%$. Thus, CMR more accurately identified cardiomyopathies among adult survivors previously undiagnosed with cardiac disease using 2DE. On the other hand, compared 3DE LVEF assessment excellent correlation was seen compared to LVEF assessment by CMR [19]. CMR, or alternatively $3 \mathrm{DE}$, should therefore be considered for LVEF confirmation in patients receiving anticancer treatment with a LVEF between 50 and $59 \%$ by $2 \mathrm{DE}$, as the cutoff value of $50 \%$ of LVEF remains the one used to decide to suspend or discontinue anticancer treatment $[30 \bullet \bullet$.

A comprehensive 2D and 3D echocardiography is sometimes a challenge in patients with breast cancer due to locoregional infiltration by the tumor, mastectomy, or breast implants. The use of contrast agents may be useful is those cases. However, CMR is recommended if an accurate LVEF calculation is still not feasible with the administration of contrast. CMR cannot be systematically recommended until now due to important limitations: a much higher cost compared with echocardiography, less accessibility, and individual limitations (i.e., claustrophobia, ferromagnetic implants).

The interest of earlier CTRCD detection, based on a better response to cardioprotective treatment when administered precociously, has prompted, as it has been the case for $2 \mathrm{DE}$, advances in CMR technologies that can detect myocardial functional changes despite still preserved LVEF. Newer CMR technologies allow the detection of subclinical myocardial dysfunction, but also offer unique information on tissue characterization, not available with other cardiovascular noninvasive imaging techniques. Some CMR techniques seem to have a higher value in the field of CTRCD (Table 1) than others. For example, myocardial edema, a sign of acute myocardial injury evaluated using T2 mapping, has not been associated until now with CTRCD [31, 33, 35, 36]. Thus, the definition of cardiotoxicity warrants reassessment in light of the advent of these newer imaging technologies.

\section{Circulating Biomarkers}

The serial measurement of serum biomarkers has been proposed to address the multiple limitations of LVEF monitoring for the early diagnosis of CTRCD. The potential of biomarkers utilization may allow for early identification of vulnerable patients in the subclinical stage of CTRCD, and may offer a more convenient, low cost method of monitoring, having predictive value in other cardiomyopathy disease states. However, the validation of a cutoff value correlating with clinically significant cardiotoxicity, and the determination of the optimal timing, frequency, and duration of testing remain the major limitations of biomarkers, restricting currently their routine use.

The most studied biomarkers are those whose values have already been validated in other cardiomyopathies, namely troponins and natriuretic peptides. Cardiac troponins are sensitive markers of myocardial injury with both troponin I (Tn-I) and $\mathrm{T}(\mathrm{Tn}-\mathrm{T})$ assays. Cardinale et al. demonstrated that the timing and degree of Tn-I rise was predictive of developing LV dysfunction, and also correlated with anthracycline cumulative dosing [43-46]. Tn-I monitoring also served effectively as risk stratification in their studied population; patients treated with high-dose chemotherapy who developed an increased in Tn-I were the ones who benefited more from the initiation of an angiotensin-converting enzyme inhibitor treatment (enalapril), preventing LVEF decrease [47]. In addition, an increase in Tn-I was associated with a 17.6 times increased risk of cardiotoxicity, and emerged as the only independent predictor of lack of recovery in LVEF despite treatment with HF therapy, which consisted of enalapril and carvedilol upbeing titrated to maximally tolerated doses used in conventional HF [48]. However, these results still need to be confirmed [49-51]. High-sensitive troponins assays may have a greater interest for the detection of subclinical myocardial damage. Sawaya et al. recently demonstrated that highsensitive Tn-I assays in combination with echocardiography strain imaging increase the accuracy of early detection and prediction of CTRCD in breast cancer patients treated with anthracyclines and trastuzumab $[22,24]$. The ASE/EACVI consensus document recommends serial Tn-I levels be drawn with a chemotherapy regimen potentially associated with type I or type II toxicities or in case of their sequential administration [11••]. In the event of encountering positive biomarkers, it is recommended to refer the patient to cardiology consultation for further evaluation and consideration of cardioprotective therapy. During chemotherapy administration, troponin monitoring is suggested before and/or $24 \mathrm{~h}$ after each chemotherapy cycle [43]. Patients with troponin elevations during therapy (as defined by the cutoffs specific to the assay platform used in the individual labs) are at a higher risk for subsequent cardiovascular events [43].

Natriuretic peptides (B-type natriuretic peptide [BNP] and N-terminal proBNP [NT-proBNP]) are cardiac hormones released from ventricular myocardium in response to increased wall stress (markers of elevated LV pressure) and standard biomarkers in clinical practice for the diagnosis and management of HF. Natriuretic peptides biomarkers have been evaluated for risk stratification prior to and during therapy for cardiotoxicity. For instance, a single baseline NT-proBNP level of $900 \mathrm{pg} / \mathrm{ml}$ or more was associated with a higher risk of death from any cause in lymphoma patients treated with chemotherapy [52]. Evidences advocating the interest of 
Table 1 Different CMR techniques for the diagnosis of subclinical cancer therapy-related myocardial lesions

\begin{tabular}{|c|c|c|c|c|c|}
\hline & $\begin{array}{l}\text { Cardiac } \\
\text { parameters }\end{array}$ & $\begin{array}{l}\text { CMR } \\
\text { techniques }\end{array}$ & Pulse sequences & General interest & Value in the field of CTRCD \\
\hline $\begin{array}{r}\text { Myocardial } \\
\text { geometry }\end{array}$ & LV mass & Cine imaging & $\begin{array}{l}\text { Balanced steady-state free } \\
\text { precession (bSSFP) }\end{array}$ & & $\begin{array}{l}\text { Indexed LV mass by CMR imaging } \\
\text { is a predictor of adverse } \\
\text { cardiovascular events [31] }\end{array}$ \\
\hline $\begin{array}{l}\text { Myocardial } \\
\text { deforma- } \\
\text { tion }\end{array}$ & Strain & $\begin{array}{l}\text { Myocardial } \\
\text { tagging }\end{array}$ & $\begin{array}{l}\text { Spatial modulation of } \\
\text { magnetization (SPAMM) }\end{array}$ & $\begin{array}{l}\text { More sensitive than LVEF } \\
\text { for the evaluation of } \\
\text { myocardial function } \\
\text { because it is a direct } \\
\text { analysis of myofibers } \\
\text { motion }\end{array}$ & $\begin{array}{l}\text { Exposure to low to moderate doses } \\
\text { of anthracyclines has been } \\
\text { associated with the early } \\
\text { deterioration of mean mid-wall } \\
\text { circumferential strain evaluated } \\
\text { with CMR [32] }\end{array}$ \\
\hline \multirow[t]{3}{*}{$\begin{array}{l}\text { Myocardial } \\
\text { composi- } \\
\text { tion }\end{array}$} & $\begin{array}{l}\text { Myocardial } \\
\text { inflammation }\end{array}$ & $\begin{array}{l}\text { Edema } \\
\text { imaging }\end{array}$ & T2 mapping & $\begin{array}{l}\text { Detection of myocardial } \\
\text { edema, a nonspecific sign } \\
\text { of acute myocardial injury }\end{array}$ & $\begin{array}{l}\text { Could be a marker of early CTRCD } \\
\text { but not yet validated }\end{array}$ \\
\hline & Diffuse fibrosis & $\begin{array}{l}\text { T1 mapping } \\
\text { or } \\
\text { extracellu- } \\
\text { lar volume } \\
\text { fraction }\end{array}$ & MOLLY & $\begin{array}{l}\text { Detection of diffuse } \\
\text { myocardial fibrosis } \\
\text { without the need for } \\
\text { comparison with normal } \\
\text { myocardium }\end{array}$ & $\begin{array}{l}\text { Association between ECV increased } \\
\text { and decreased exercise capacity } \\
\text { and LV mass to LV volume ratio } \\
{[33,34] \text {, diastolic dysfunction }} \\
\text { [35] and LVEF decline [36] }\end{array}$ \\
\hline & Focal fibrosis & $\begin{array}{l}\text { Late } \\
\text { gadolinium } \\
\text { enhance- } \\
\text { ment } \\
\text { imaging }\end{array}$ & & $\begin{array}{l}\text { Delineates regional } \\
\text { myocardial scar or } \\
\text { fibrosis, helping } \\
\text { elucidating etiologies of } \\
\text { cardiomyopathies }\end{array}$ & $\begin{array}{l}\text { Controversial No typical pattern of } \\
\text { LGE, suggesting patchy } \\
\text { myocarditis }[23,31,34,35 \text {, } \\
37-39] \\
\text { Less reported than diffuse fibrosis }\end{array}$ \\
\hline $\begin{array}{l}\text { Myocardial } \\
\text { perfusion } \\
\text { reserve }\end{array}$ & $\begin{array}{l}\text { Coronary flow } \\
\text { reserve } \\
\text { (epicardial } \\
\text { circulation } \\
\text { and } \\
\text { microcircula- } \\
\text { tion) }\end{array}$ & $\begin{array}{l}\text { Stress } \\
\quad \text { perfusion } \\
\text { imaging }\end{array}$ & $\begin{array}{l}\text { T1-weighted ECG-gated single } \\
\text { shot 2D sequence With an } \\
\text { intravenous dilatator or } \\
\text { inotropic agent Require } \\
\text { contrast agent gadolinium }\end{array}$ & $\begin{array}{l}\text { Perfusion CMR is superior } \\
\text { and more sensitive than } \\
\text { SPECT }[40,41]\end{array}$ & $\begin{array}{l}\text { Chest radiation accelerate epicardial } \\
\text { and microvascular disease but } \\
\text { very few studies have used stress } \\
\text { CMR in cancer survivors }[38,42]\end{array}$ \\
\hline
\end{tabular}

natriuretic peptides monitoring during the course of chemotherapy exist [53-56]. A persistently increased NT-proBNP (early after high-dose chemotherapy administration) is predictive of subsequent LV dysfunction during follow-up [53, 55]. However, other studies found no correlation between natriuretic peptide increase and development of cardiac dysfunction in patients receiving anthracycline-based chemotherapy [57]. Those results are explained by the difficulty in interpreting these biomarkers among heterogeneous populations (small number of patients, various different types of malignancies, varying chemotherapeutic regimens), using different natriuretic peptides (BNP, NT-proBNP, atrial natriuretic peptide [ANP], NT-proANP), different laboratory methods, cutoffs, sampling time, and differing follow-up duration and endpoints. Furthermore, BNP and NT-proBNP are makers of wall stress rather than myocardial injury. Elevations during cancer therapy may not be as specific as troponins elevations [58].

Finally, many emerging biomarkers, exploring different potential mechanisms of cardiotoxicity, have been proposed for monitoring of cardiotoxicity in recent studies [59, 60]. In a multicenter cohort of 78 patients with breast cancer undergoing doxorubicin and trastuzumab therapy, 8 potential relevant biomarkers (high-sensitive Tn-I, high-sensitivity C-reactive protein, NT-proBNP, growth differentiation factor-15, myeloperoxidase [MPO], placental growth factor, soluble fms-like tyrosine kinase receptor-1 and galectin-3) were evaluated at baseline and every 3 months over a follow-up period of 15 months. The risk of cardiotoxicity was not significantly associated with baseline levels of any of these biomarkers. However, the early changes in Tn-I and MPO, the combination of increasing biomarkers and finally the persistence of an increase in MPO beyond 3 months, were predictors of cardiotoxicity risk over the duration of doxorubicin/ trastuzumab therapy $[61,62]$.

\section{Multimodality Baseline and Surveillance Strategies}

An approach that integrates circulating biomarkers and noninvasive cardiac imaging parameters for baseline evaluation and surveillance strategy may provide additive value in predicting cardiotoxicity. This conceptual approach requires close multidisciplinary collaboration of the oncologist and the cardiologist in interpreting and reacting to the results of these findings. 


\section{Baseline Cardiac Evaluation}

The baseline cardiac evaluation is very important and requires standardization. The authors propose that such an evaluation should be considered in every patient receiving chemotherapy agents associated with known or suspected cardiotoxicity. It should also be routinely performed in patients with a documented pre-existing or acquired cardiomyopathy, risk factors of cardiac disease, $>65$ years old and scheduled high-dose type I agents or combined regimens with type I and type II agents $[11 \bullet \bullet]$. Baseline global cardiovascular risk assessment should also be included, as they have been demonstrated to be risk factors of CTRCD [30••]. The baseline cardiac evaluation also includes a thorough medical history, physical examination, electrocardiogram, and LVEF quantification. Indeed, LVEF remains an important screening tool. 2DE, 3DE, and CMR quantification of LVEF show good correlation [63] but absolute values can differ. Thus, LVEF obtained from different imaging modalities may not be interchangeable [64]. In routine practice, a single technique ideally should be used for baseline assessment and follow-up studies during and after cancer therapy $[11 \bullet \cdot]$. CMR should always be considered when 2DE-derived LVEF is suboptimally visualized and/or serially noted to have discrepant findings, particularly in patients with lower limit of normal LVEF (2DE has been known to overestimate the LVEF in this population compared to CMR) [18]. Borderline low baseline LVEF holds a confirmed predictive value to identify among women treated for breast cancer those at risk of CTRCD $[65,66]$. However, a normal baseline LVEF assessment alone does not formally exclude the occurrence of chemotherapy-induced cardiac events: congestive HF has also been reported in 2.1 and $4.2 \%$ of patients with a baseline LVEF of $\geq 65 \%$ and $55-64 \%$, respectively [65]. If feasible (good image quality, software available), baseline 2DE should include a baseline assessment of GLS [11••]. Finally, measurement of cardiac biomarkers - troponins and/ or natriuretic peptides - may be considered at baseline although evidence supporting this strategy is still lacking $[11 \bullet \bullet, 67 \bullet \bullet]$.

\section{Cardiac Monitoring During Cardiotoxic Cancer Therapy}

At present, serial monitoring of LVEF remains the most frequently used parameter for detecting CTRCD. Concerning type I agents, the recommended timing for surveillance is at the completion of therapy, particularly when the patient has an increased risk for cardiotoxicity or consecutive treatment with potentially cardiotoxic targeted therapies will follow [11••, $67 \bullet \bullet]$. For regimens including cumulative total doxorubicin (or equivalent) doses $>240 \mathrm{mg} / \mathrm{m}^{2}$, an evaluation before each additional $50 \mathrm{mg} / \mathrm{m}^{2}$ is strongly recommended. Additional monitoring is finally mentioned 6 months after the completion of anthracycline. For type II agents, LVEF assessment every
3 months is generally recommended and once after completion of treatment $[67 \bullet \bullet$.

However, an important additive value of effective cardiac surveillance during chemotherapy is the detection of subclinical LV dysfunction. To meet this objective, as discussed above, LVEF assessment alone is insufficient. The authors propose a strategy that include, in addition to LVEF assessment, the GLS calculation and the baseline chosen biomarker measurement, all for prospective comparison with baseline data. Indeed, addition of monitored parameters via a multimodality surveillance strategy increases sensitivity of subclinical LV dysfunction detection [61, 62]. However, only a few studies have explored the value of such a combined approach for the diagnosis and risk stratification of CTRCD (Table 2, Fig. 3). Fallah-Rad et al. conducted the first multimodality surveillance strategy, combining biomarkers (troponin T, CRP, and BNP) with imaging (TTE and CMR) in breast cancer patients treated in the adjuvant setting by anthracyclines and trastuzumab [23]. Biomarkers were not associated with any prognostic value, along with LVEF assessment, but Doppler measurement of s', GLS, and radial strain parameters were able to identify at 3 months followup the patients that developed CTRCD at 6 months. In this study, CMR, performed at baseline and at 12 months, documented an increase in LV volumes, a decrease in LVEF, and a late gadolinium enhancement in the LV lateral wall in the CTRCD group. Sawaya et al. studied a very similar population with similar biomarkers [22]. NT-proBNP was not associated with any predictive value but high-sensitive Tn-I at 3 months appeared as an independent predictor of cardiotoxicity at 6 months. A combination of myocardial longitudinal strain and high-sensitive Tn-I allowed the identification of subtle myocardial alterations and was predictive of subsequent LVEF reduction during the routine surveillance of patients receiving trastuzumab after anthracyclines. The high negative predictive value of the two tests also allowed the confident exclusion of cardiotoxicity 3 months later. In a longer follow-up of 15 months, in a similar breast cancer population, a GLS value of $<19 \%$ and high-sensitive Tn-I level of $\geq 30 \mathrm{pg} / \mathrm{ml}$ measured after anthracycline administration and before taxanes and trastuzumab infusion were predictive of subsequent development of CTRCD [24]. The troponin levels added prognostic value to GLS: if both were abnormal, the specificity for the prediction of CTRCD increased from 73 to $93 \%$. If both were normal, the negative predictive value increases to $91 \%$ [24].

These studies, although limited and small in scope, support an integrated approach for cardiac surveillance and subclinical LV dysfunction detection in the ASE/EACVI expert consensus statement $[11 \bullet \cdot$. A relative percentage reduction of GLS, with the selected cutoff value of $>15 \%$, is very likely to be abnormal, whereas a change $<8 \%$ seems not clinically significant. In regards to biomarkers, the consensus document report 
Table 2 Comparison of studies available about multimodality strategies during anticancer treatment surveillance

\begin{tabular}{|c|c|c|c|c|c|c|c|}
\hline & $\begin{array}{l}\text { Cancer type, } \\
\text { chemotherapy } \\
\text { regimens }\end{array}$ & $\begin{array}{l}\text { Multimodality } \\
\text { strategy }\end{array}$ & $\begin{array}{l}\text { Number } \\
\text { of } \\
\text { centers }\end{array}$ & $\begin{array}{l}\text { Number } \\
\text { of } \\
\text { patients }\end{array}$ & Definition of CTRCD & $\begin{array}{l}\text { Timing of } \\
\text { surveillance } \\
\text { strategy }\end{array}$ & Results \\
\hline $\begin{array}{c}\text { Sawaya } \\
{[22]}\end{array}$ & $\begin{array}{l}\text { Breast cancer, } \\
\text { adjuvant } \\
\mathrm{A}+\mathrm{T}\end{array}$ & $\begin{array}{l}\text { Circulating } \\
\text { biomarkers } \\
\text { (NT-proBNP, } \\
\text { hsTn-I) TTE }\end{array}$ & 4 & 43 & $\begin{array}{l}\text { Reduction of LVEF of } \geq 5 \% \text { to } \\
<55 \% \text { with HF symptoms } \\
\text { or asymptomatic reduction } \\
\text { of LVEF of } \geq 10 \text { to }<55 \%\end{array}$ & $\begin{array}{l}\text { Baseline } \\
3 \text { months } \\
6 \text { months }\end{array}$ & $\begin{array}{l}\text { - } 9 / 43(21 \%) \text { met the criteria of } \\
\text { CTRCD } \\
\text { - hsTn- } I>0.015 \mathrm{mcg} / \mathrm{l} \text { at } \\
3 \text { months }(p<0.02) \text { and } \\
\text { decrease in GLS at } 3 \text { months } \\
(p<0.02) \text { are independent } \\
\text { predictors of CTRCD at } \\
6 \text { months } \\
\text { - patients who either demonstrate } \\
\text { a }>10 \% \text { decrease in GLS or } \\
\text { elevation in hsTn-I at } 3 \text { months } \\
\text { have a ninefold increase in risk } \\
\text { for CTRCD at } 6 \text { months }\end{array}$ \\
\hline $\begin{array}{l}\text { Fallah-Rad } \\
\quad \text { [23] }\end{array}$ & $\begin{array}{l}\text { Breast cancer, } \\
\text { adjuvant } \\
\mathrm{A}+\mathrm{T}\end{array}$ & $\begin{array}{l}\text { Circulating } \\
\text { biomarkers } \\
\text { (NT-proBNP, } \\
\text { Tn-T, CRP) } \\
\text { TTE CMR }\end{array}$ & 1 & 42 & $\begin{array}{l}\text { Reduction in LVEF of }>10 \text { to } \\
<55 \% \text { with HF symptoms }\end{array}$ & $\begin{array}{l}\text { Baseline } \\
\text { Before T } \\
3 \text { months } \\
6 \text { months } \\
9 \text { months } \\
12 \text { months }\end{array}$ & $\begin{array}{l}\text { - } 10 / 42(25 \%) \text { met the criteria of } \\
\text { CTRCD } \\
\text { - no predictive value of } \\
\text { biomarkers } \\
\text { - lateral s'<6 cm/s at } 3 \text { months is } \\
\text { predictive of CTRCD: } \\
\text { sensitivity } 93 \% \text {, specificity } \\
99 \%, \text { PPV } 96 \%, \text { NPV } 98 \% \\
\text { - a } 2 \% \text { reduction of GLS at } \\
3 \text { months is predictive of } \\
\text { CTRCD: sensitivity } 79 \% \text {, } \\
\text { specificity } 82 \%, \text { PPV } 60 \% \text {, } \\
\text { NPV } 92 \% \\
\text { - CMR: not studied as predictive } \\
\text { parameter }\end{array}$ \\
\hline $\begin{array}{c}\text { Sawaya } \\
{[24]}\end{array}$ & $\begin{array}{l}\text { Breast cancer, } \\
\text { adjuvant } \\
\mathrm{A}+\mathrm{Tx}+ \\
\mathrm{T}\end{array}$ & $\begin{array}{l}\text { Circulating } \\
\text { biomarkers } \\
\text { (NT-proBNP, } \\
\text { hsTn-I, ST2) } \\
\text { TTE }\end{array}$ & 4 & 81 & $\begin{array}{l}\text { Reduction of LVEF of } \geq 5 \% \text { to } \\
<55 \% \text { with HF symptoms } \\
\text { or asymptomatic reduction } \\
\text { of LVEF of } \geq 10 \% \text { to }<55 \%\end{array}$ & $\begin{array}{l}\text { Baseline } \\
3 \text { months } \\
6 \text { months } \\
9 \text { months } \\
12 \text { months } \\
15 \text { months }\end{array}$ & $\begin{array}{l}\text { - } 26 / 81(32 \%) \text { met the criteria of } \\
\text { CTRCD } \\
\text { - GLS < 19\% is the only } \\
\text { independent predictor of } \\
\text { CTRCD ( } p=0.0003) \\
\text { - hsTn-I measurement combined } \\
\text { with GLS increase the } \\
\text { sensitivity of the biomarkers } \\
\text { from } 74 \% \text { to } 87 \%, \text { NPP } 91 \%\end{array}$ \\
\hline
\end{tabular}

$A$ anthracyclines, $C M R$ cardiac magnetic resonance, $C T R C D$ cancer therapeutic-related cardiac dysfunction, $G L S$ global longitudinal strain, $H F$ heart failure, $L V E F$ left ventricular ejection fraction, $N T$-proBNP N-terminal pro-brain natriuretic peptide, $N P V$ negative predictive value, $P P V$ positive predictive value, $T$ trastuzumab, $T n-T$ troponin T, $T T E$ transthoracic echocardiography, $T x$ taxanes, $h s T n-I$ high-sensitive troponin I

that troponins elevation, measured before and/or $24 \mathrm{~h}$ after chemotherapy infusion, is associated with a higher risk of cardiovascular events and cardiology consultation should be considered. No specific cutoff value is given, but one must refer to the cutoffs specific to the essay platform used in individual labs.

\section{Proposed Multimodality Imaging Strategies}

Cardiovascular complications can be a concerning issue during the course of cancer therapy, as cardiac side effects adversely affect oncological therapy and cancer prognosis.
Current cardiac surveillance strategies are limited due to from a lack of standardization as well as studies validating their approaches. Patients scheduled to receive high dose of type I agents, type II agents, or sequential chemotherapy with type I and type II agents require a thorough, systematic, and consistent approach to their cardiac evaluation which has yet to be determined. Secondly, current CTRCD definition is based on a decrease of the LVEF [11 ••], even though LVEF assessment alone is likely insufficient, as end stage myocardial insult is likely present by the time this manifests, particularly in type I cardiotoxicity. While cancer survival rates have increased due to significant advances made in treatment, efforts made to reduce short- and long-term cardiotoxicity should be a priority 

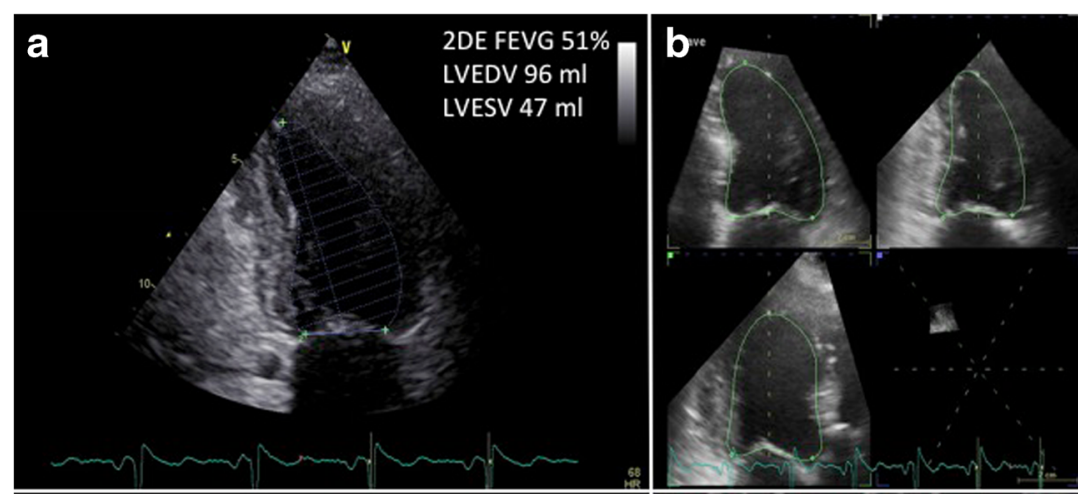

3DE FEVG 51\%

LVEDV $118 \mathrm{ml}$

LVESV $58 \mathrm{ml}$

SV $60 \mathrm{ml}$
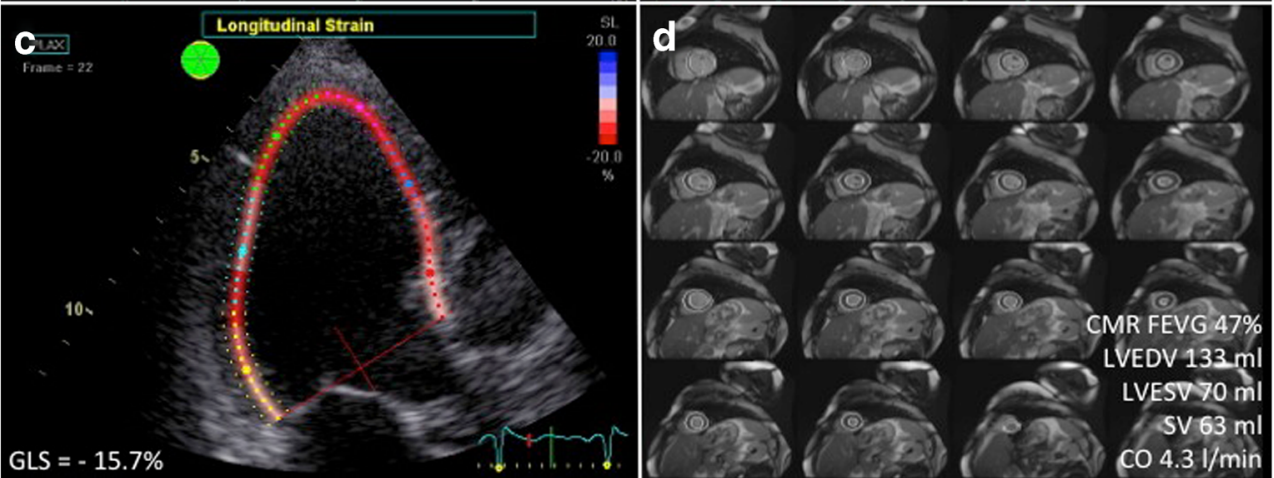

Fig. 3 Forty-six-year-old woman diagnosed with right breast cancer (invasive ductal carcinoma) with lymph node involvement. The anatomopathology analysis revealed triple negative carcinoma. Her previous medical history included treated hypertension, where she was taking olmesartan $40 \mathrm{mg}$ daily and bisoprolol $2.5 \mathrm{mg}$ daily, and type II diabetes mellitus. She received neo-adjuvant chemotherapy (4 regimens of epirubicin-cyclophosphamide followed by 12 weekly carboplatinpaclitaxel) before undergoing right mastectomy and axillary lymph node dissection. Histopathology revealed HER-2 positive invasive ductal carcinoma, warranting adjuvant trastuzumab. Her baseline LVEF was $65 \%$. Post anthracycline 2D LVEF was $51 \%$ (a), confirmed at

3 weeks, corresponding to the definition of CTRCD. 3D LVEF was also calculated at $51 \%$ (b), GLS was- $15.7 \%$ (c), and CMR LVEF $47 \%$ (d). Trastuzumab was initiated with close monitoring of LVEF (every 3 weeks) using multimodality approach (2DE, 3DE, and GLS) and with cardioprotective treatment titrated to the maximal tolerated dosage. $C M R$ cardiac magnetic resonance, $C O$ cardiac output, $C T R C D$ cancer therapeutic-related cardiac dysfunction, $G L S$ global longitudinal strain, $L V E D V$ left ventricular end diastolic volume, $L V E F$ left ventricular ejection fraction, $L V E S V$ left ventricular end systolic volume, $S V$ stroke volume

in ongoing research efforts [68]. Developing surveillance strategies allowing an earlier detection of CTRCD are of utmost importance, and standardized care plans incorporating multimodality imaging may potentially meet the objective of earlier detection of myocardial dysfunction, and can lead to earlier initiation of cardioprotective agents (beta blockers, angiotensin-converting enzyme inhibitors, or angiotensin II receptor blockers).

First, any strategy will depend on a standardized baseline cardiac assessment. Concerning the cardiac imaging aspects, the gold standard is TTE. Precise baseline LVEF measurement, using $2 \mathrm{DE}$ or preferably $3 \mathrm{DE}$, is fundamental and GLS assessment is desirable. Biomarkers dosage is also suggested. Any abnormality of these three parameters should lead to multidisciplinary discussion between cardiologist and oncologist in determining surveillance frequency for cardiotoxicity, and consideration of implementing cardioprotective drugs, and the choice of continuing or altering cancer treatment to be at the discretion of the oncologist. Baseline CMR is indicated if suboptimal echocardiographic images are obtained, or of a borderline abnormal LVEF or discrepant readings on serial studies.

The timing of surveillance using cardiac imaging and biomarkers should be established based on cardiotoxicity profile of the chemotherapy regimen, ideally personalized to the patient baseline cardiovascular risk $[67 \bullet \bullet, 69]$. The goals of treatment, whether it be adjuvant, neo-adjuvant, or palliative chemotherapy, also should determine the extent of cardiotoxicity monitoring with ongoing weighing of risks and benefits of continuing treatments if LV dysfunction occurs.

CTRCD definition is based on the LVEF evaluation and this parameter should normally be numerically reported at each evaluation. As LVEF obtained from TTE (2DE, 3DE) and CMR may not be interchangeable, the same imaging technique as the one chosen for baseline evaluation should be used for surveillance. In the same time, the follow-up studies should also report any evidence of subclinical LV dysfunction. Available validated tools are GLS and biomarkers. GLS has been considered to be accurate and prognostic in detecting subclinical cardiotoxicity in patients receiving cardiotoxic 
anticancer agents [21-27]. Cutoffs value for relative percentage reduction during surveillance which are suggestive of clinically significant cardiotoxicity has been proposed in the ASE/EACVI expert consensus document [11 ••], although further studies are needed to validate these findings.

In regards to biomarkers, troponin levels appear to have the best predictive value in detecting cardiotoxicity monitoring, with the timing and the degree of the rise correlated with risk of CTRCD [43-46]. Troponin elevations during treatment also identify a high risk cohort of cancer patients who may benefit from early cardioprotective medication [47]. Natriuretic peptides are strong predictors of elevated LV pressure and HF. For subclinical LV dysfunction detection during cancer therapy, data is inconsistent but it appears that the persistence of NT-proBNP elevation during follow-up identifies patients at higher risk of LVEF decline [53, 55].

\section{Conclusions}

In conclusion, a multimodality surveillance strategy, utilizing both imaging and biomarkers, is ideal in accurately detecting subclinical LV dysfunction induced by cardiotoxic chemotherapy. While advances in imaging technology have allowed for more precise assessment of cardiac function, CTRCD criteria of LV dysfunction have not been updated to include parameters such as subclinical dysfunction, or how to accurately assess LVEF using different imaging modalities. While the most optimal approach remains to be seen and requires prospective study, cancer patients have the potential to benefit from earlier detection of cardiotoxicity and intervention, allowing them to potentially continue with lifesaving treatments.

\section{Compliance with Ethical Standards}

Conflict of Interest Marie Moonen, Cécile Oury, and Patrizio Lancellotti declare they have no conflict of interest.

Human and Animal Rights and Informed Consent This article does not contain any studies with human or animal subjects performed by any of the authors.

\section{References}

Papers of particular interest, published recently, have been highlighted as:

- Of importance

•- Of major importance

1. Moslehi JJ. Cardiovascular toxic effects of targeted cancer therapies. N Engl J Med. 2016;375(15):1457-67. doi:10.1056/
NEJMra1100265. Recent review on the cardiovascular toxic effects associated with new targeted cancer therapies.

2. Witteles RM, Bosch X. Myocardial protection during cardiotoxic chemotherapy. Circulation. 2015;132(19):1835-45. doi:10.1161/ circulationaha.114.010486.

3. Lancellotti P, Moonen M, Jerusalem G. Predicting reversibility of anticancer drugs-related cardiac dysfunction: a new piece to the routine use of deformation imaging. Echocardiography. 2016;33(4):504-9. doi:10.1111/echo.13187.

4. Fanous I, Dillon P. Cancer treatment-related cardiac toxicity: prevention, assessment and management. Med Oncol. 2016;33(8):84. doi:10.1007/s12032-016-0801-5.

5. Ewer MS, Vooletich MT, Durand JB, Woods ML, Davis JR, Valero $\mathrm{V}$, et al. Reversibility of trastuzumab-related cardiotoxicity: new insights based on clinical course and response to medical treatment. J Clin Oncol. 2005;23(31):7820-6. doi:10.1200/JCO.2005.13.300.

6. Felker GM, Thompson RE, Hare JM, Hruban RH, Clemetson DE, Howard DL, et al. Underlying causes and long-term survival in patients with initially unexplained cardiomyopathy. N Engl J Med. $2000 ; 342(15): 1077-84$. doi:10.1056/ nejm200004133421502.

7. Ghosh N, Hilton J. Orthotopic heart transplantation and mechanical circulatory support in cancer survivors: challenges and outcomes. J Oncol. 2015;2015:232607. doi:10.1155/2015/232607.

8. Thavendiranathan P, Abdel-Qadir H, Fischer HD, Camacho X, Amir E, Austin PC, et al. Breast cancer therapy-related cardiac dysfunction in adult women treated in routine clinical practice: a population-based cohort study. J Clin Oncol. 2016;34(19):2239 46. doi:10.1200/jco.2015.65.1505.

9. Cardinale D, Colombo A, Lamantia G, Colombo N, Civelli M, De Giacomi G, et al. Anthracycline-induced cardiomyopathy: clinical relevance and response to pharmacologic therapy. J Am Coll Cardiol. 2010;55(3):213-20. doi:10.1016/j.jacc.2009.03.095.

10. Gottdiener JS, Mathisen DJ, Borer JS, Bonow RO, Myers CE, Barr LH, et al. Doxorubicin cardiotoxicity: assessment of late left ventricular dysfunction by radionuclide cineangiography. Ann Intern Med. 1981;94(4):430-5.

11.•P Plana JC, Galderisi M, Barac A, Ewer MS, Ky B, Scherrer-Crosbie $\mathrm{M}$, et al. Expert consensus for multimodality imaging evaluation of adult patients during and after cancer therapy: a report from the American Society of Echocardiography and the European Association of Cardiovascular Imaging. Eur Heart J Cardiovasc Imaging. 2014;15(10):1063-93. doi:10.1093/ehjci/jeu192. Outlines intersociety recommendations for imaging of adult cancer patients and survivors.

12. Otterstad JE, Froeland G, St John Sutton M, Holme I. Accuracy and reproducibility of biplane two-dimensional echocardiographic measurements of left ventricular dimensions and function. Eur Heart J. 1997;18(3):507-13.

13. Thavendiranathan P, Grant AD, Negishi T, Plana JC, Popovic ZB, Marwick TH. Reproducibility of echocardiographic techniques for sequential assessment of left ventricular ejection fraction and volumes: application to patients undergoing cancer chemotherapy. J Am Coll Cardiol. 2013;61(1):77-84. doi:10.1016/j.jacc.2012.09. 035.

14. Senior R, Becher H, Monaghan M, Agati L, Zamorano J, Vanoverschelde JL, et al. Contrast echocardiography: evidencebased recommendations by European Association of Echocardiography. Eur J Echocardiogr. 2009;10(2):194-212. doi: 10.1093/ejechocard/jep005.

15. Mulvagh SL, Rakowski H, Vannan MA, Abdelmoneim SS, Becher $\mathrm{H}$, Bierig SM, et al. American Society of Echocardiography consensus statement on the clinical applications of ultrasonic contrast agents in echocardiography. J Am Soc Echocardiogr. 2008;21(11): 1179-201. doi:10.1016/j.echo.2008.09.009. 
16. Jenkins C, Chan J, Hanekom L, Marwick TH. Accuracy and feasibility of online 3-dimensional echocardiography for measurement of left ventricular parameters. J Am Soc Echocardiogr. 2006;19(9): 1119-28. doi:10.1016/j.echo.2006.04.002.

17. Jenkins C, Moir S, Chan J, Rakhit D, Haluska B, Marwick TH. Left ventricular volume measurement with echocardiography: a comparison of left ventricular opacification, three-dimensional echocardiography, or both with magnetic resonance imaging. Eur Heart J. 2009;30(1):98-106. doi:10.1093/eurheartj/ehn484.

18. Armstrong GT, Plana JC, Zhang N, Srivastava D, Green DM, Ness $\mathrm{KK}$, et al. Screening adult survivors of childhood cancer for cardiomyopathy: comparison of echocardiography and cardiac magnetic resonance imaging. J Clin Oncol. 2012;30(23):2876-84. doi:10. 1200/jco.2011.40.3584.

19. Walker J, Bhullar N, Fallah-Rad N, Lytwyn M, Golian M, Fang T, et al. Role of three-dimensional echocardiography in breast cancer: comparison with two-dimensional echocardiography, multiplegated acquisition scans, and cardiac magnetic resonance imaging. J Clin Oncol. 2010;28(21):3429-36. doi:10.1200/jco.2009.26. 7294.

20. Mignot A, Donal E, Zaroui A, Reant P, Salem A, Hamon C, et al. Global longitudinal strain as a major predictor of cardiac events in patients with depressed left ventricular function: a multicenter study. J Am Soc Echocardiogr. 2010;23(10):1019-24. doi:10. 1016/j.echo.2010.07.019.

21. Ho E, Brown A, Barrett P, Morgan RB, King G, Kennedy MJ, et al. Subclinical anthracycline- and trastuzumab-induced cardiotoxicity in the long-term follow-up of asymptomatic breast cancer survivors: a speckle tracking echocardiographic study. Heart. 2010;96(9):701-7. doi:10.1136/hrt.2009.173997.

22. Sawaya H, Sebag IA, Plana JC, Januzzi JL, Ky B, Cohen V, et al. Early detection and prediction of cardiotoxicity in chemotherapytreated patients. Am J Cardiol. 2011;107(9):1375-80. doi:10.1016/ j.amjcard.2011.01.006.

23. Fallah-Rad N, Walker JR, Wassef A, Lytwyn M, Bohonis S, Fang $\mathrm{T}$, et al. The utility of cardiac biomarkers, tissue velocity and strain imaging, and cardiac magnetic resonance imaging in predicting early left ventricular dysfunction in patients with human epidermal growth factor receptor II-positive breast cancer treated with adjuvant trastuzumab therapy. J American Coll Cardiol. 2011;57(22): 2263-70. doi:10.1016/j.jacc.2010.11.063.

24. Sawaya H, Sebag IA, Plana JC, Januzzi JL, Ky B, Tan TC, et al. Assessment of echocardiography and biomarkers for the extended prediction of cardiotoxicity in patients treated with anthracyclines, taxanes, and trastuzumab. Circ Cardiovasc Imaging. 2012;5(5): 596-603. doi:10.1161/circimaging.112.973321.

25. Stoodley PW, Richards DA, Boyd A, Hui R, Harnett PR, Meikle $\mathrm{SR}$, et al. Left ventricular systolic function in HER2/neu negative breast cancer patients treated with anthracycline chemotherapy: a comparative analysis of left ventricular ejection fraction and myocardial strain imaging over 12 months. Eur J Cancer. 2013;49(16): 3396-403. doi:10.1016/j.ejca.2013.06.046.

26. Negishi K, Negishi T, Hare JL, Haluska BA, Plana JC, Marwick $\mathrm{TH}$. Independent and incremental value of deformation indices for prediction of trastuzumab-induced cardiotoxicity. J Am Soc Echocardiogr. 2013;26(5):493-8. doi:10.1016/j.echo.2013.02.008.

27. Fei HW, Ali MT, Tan TC, Cheng KH, Salama L, Hua L, et al. Left ventricular global longitudinal strain in HER-2 + breast cancer patients treated with anthracyclines and trastuzumab who develop cardiotoxicity is associated with subsequent recovery of left ventricular ejection fraction. Echocardiography. 2016;33(4):519-26. doi:10.1111/echo.13168.

28. Grothues F, Smith GC, Moon JC, Bellenger NG, Collins P, Klein $\mathrm{HU}$, et al. Comparison of interstudy reproducibility of cardiovascular magnetic resonance with two-dimensional echocardiography in normal subjects and in patients with heart failure or left ventricular hypertrophy. Am J Cardiol. 2002;90(1):29-34.

29. Hundley WG, Bluemke DA, Finn JP, Flamm SD, Fogel MA, Friedrich MG, et al. ACCF/ACR/AHA/NASCI/SCMR 2010 expert consensus document on cardiovascular magnetic resonance: a report of the American College of Cardiology Foundation Task Force on expert consensus documents. Circulation. 2010;121(22): 2462-508. doi:10.1161/CIR.0b013e3181d44a8f.

30.• Curigliano G, Cardinale D, Suter T, Plataniotis G, de Azambuja E, Sandri MT, et al. Cardiovascular toxicity induced by chemotherapy, targeted agents and radiotherapy: ESMO Clinical Practice Guidelines. Ann Oncol. 2012;23(Suppl 7):vii155-66. doi:10. 1093/annonc/mds293. European society for medical oncology practice guidelines.

31. Neilan TG, Coelho-Filho OR, Pena-Herrera D, Shah RV, JeroschHerold M, Francis SA, et al. Left ventricular mass in patients with a cardiomyopathy after treatment with anthracyclines. Am J Cardiol. 2012;110(11):1679-86. doi:10.1016/j.amjcard.2012.07.040.

32. Drafts BC, Twomley KM, D'Agostino R Jr, Lawrence J, Avis N, Ellis LR, et al. Low to moderate dose anthracycline-based chemotherapy is associated with early noninvasive imaging evidence of subclinical cardiovascular disease. JACC Cardiovasc Imaging. 2013;6(8):877-85. doi:10.1016/j.jcmg.2012.11.017.

33. Tham EB, Haykowsky MJ, Chow K, Spavor M, Kaneko S, Khoo NS, et al. Diffuse myocardial fibrosis by T1-mapping in children with subclinical anthracycline cardiotoxicity: relationship to exercise capacity, cumulative dose and remodeling. J Cardiovasc Magn Reson. 2013;15:48. doi:10.1186/1532-429x-15-48.

34. Cheung YF, Lam WW, Ip JJ, Cheuk DK, Cheng FW, Yang JY, et al. Myocardial iron load and fibrosis in long term survivors of childhood leukemia. Pediatr Blood Cancer. 2015;62(4):698-703. doi:10. $1002 /$ pbc. 25369.

35. Neilan TG, Coelho-Filho OR, Shah RV, Feng JH, Pena-Herrera D, Mandry D, et al. Myocardial extracellular volume by cardiac magnetic resonance imaging in patients treated with anthracyclinebased chemotherapy. Am J Cardiol. 2013;111(5):717-22. doi:10. 1016/j.amjcard.2012.11.022.

36. Jordan JH, D'Agostino RB Jr, Hamilton CA, Vasu S, Hall ME, Kitzman DW, et al. Longitudinal assessment of concurrent changes in left ventricular ejection fraction and left ventricular myocardial tissue characteristics after administration of cardiotoxic chemotherapies using $\mathrm{T} 1$-weighted and $\mathrm{T} 2$-weighted cardiovascular magnetic resonance. Circ Cardiovasc Imaging. 2014;7(6):872-9. doi:10. 1161/circimaging. 114.002217.

37. Ylanen K, Poutanen T, Savikurki-Heikkila P, Rinta-Kiikka I, Eerola A, Vettenranta K. Cardiac magnetic resonance imaging in the evaluation of the late effects of anthracyclines among long-term survivors of childhood cancer. J Am Coll Cardiol. 2013;61(14):153947. doi:10.1016/j.jacc.2013.01.019.

38. de Ville de Goyet M, Brichard B, Robert A, Renard L, Veyckemans F, Vanhoutte L, et al. Prospective cardiac MRI for the analysis of biventricular function in children undergoing cancer treatments. Pediatr Blood Cancer. 2015;62(5):867-74. doi:10.1002/pbc.25381.

39. Lunning MA, Kutty S, Rome ET, Li L, Padiyath A, Loberiza F, et al. Cardiac magnetic resonance imaging for the assessment of the myocardium after doxorubicin-based chemotherapy. Am J Clin Oncol. 2015;38(4):377-81. doi:10.1097/COC.0b013e31829e19be.

40. Greenwood JP, Maredia N, Younger JF, Brown JM, Nixon J, Everett CC, et al. Cardiovascular magnetic resonance and singlephoton emission computed tomography for diagnosis of coronary heart disease (CE-MARC): a prospective trial. Lancet. 2012;379(9814):453-60. doi:10.1016/s0140-6736(11)61335-4.

41. Greenwood JP, Motwani M, Maredia N, Brown JM, Everett CC, Nixon J, et al. Comparison of cardiovascular magnetic resonance and single-photon emission computed tomography in women with suspected coronary artery disease from the Clinical Evaluation of 
Magnetic Resonance Imaging in Coronary Heart Disease (CEMARC) Trial. Circulation. 2014;129(10):1129-38. doi:10.1161/ circulationaha.112.000071.

42. Haykowsky MJ, Mackey JR, Thompson RB, Jones LW, Paterson DI. Adjuvant trastuzumab induces ventricular remodeling despite aerobic exercise training. Clin Cancer Res. 2009;15(15):4963-7. doi:10.1158/1078-0432.ccr-09-0628.

43. Cardinale D, Sandri MT, Martinoni A, Tricca A, Civelli M, Lamantia $\mathrm{G}$, et al. Left ventricular dysfunction predicted by early troponin I release after high-dose chemotherapy. J Am Coll Cardiol. 2000;36(2):517-22.

44. Sandri MT, Cardinale D, Zorzino L, Passerini R, Lentati P, Martinoni A, et al. Minor increases in plasma troponin I predict decreased left ventricular ejection fraction after high-dose chemotherapy. Clin Chem. 2003;49(2):248-52.

45. Cardinale D, Sandri MT, Martinoni A, Borghini E, Civelli M, Lamantia G, et al. Myocardial injury revealed by plasma troponin I in breast cancer treated with high-dose chemotherapy. Ann Oncol. 2002;13(5):710-5

46. Cardinale D, Sandri MT, Colombo A, Colombo N, Boeri M, Lamantia G, et al. Prognostic value of troponin I in cardiac risk stratification of cancer patients undergoing high-dose chemotherapy. Circulation. 2004;109(22):2749-54. doi:10.1161/01.cir. 0000130926.51766.cc.

47. Cardinale D, Colombo A, Sandri MT, Lamantia G, Colombo N, Civelli M, et al. Prevention of high-dose chemotherapy-induced cardiotoxicity in high-risk patients by angiotensin-converting enzyme inhibition. Circulation. 2006;114(23):2474-81. doi:10.1161/ circulationaha. 106.635144.

48. Cardinale D, Colombo A, Torrisi R, Sandri MT, Civelli M, Salvatici $\mathrm{M}$, et al. Trastuzumab-induced cardiotoxicity: clinical and prognostic implications of troponin I evaluation. J Clin Oncol. 2010;28(25): 3910-6. doi:10.1200/jco.2009.27.3615.

49. Soker M, Kervancioglu M. Plasma concentrations of NT-pro-BNP and cardiac troponin-I in relation to doxorubicin-induced cardiomyopathy and cardiac function in childhood malignancy. Saudi Med J. 2005;26(8):1197-202.

50. Mathew P, Suarez W, Kip K, Bayar E, Jasty R, Matloub Y, et al. Is there a potential role for serum cardiac troponin I as a marker for myocardial dysfunction in pediatric patients receiving anthracycline-based therapy? A pilot study. Cancer Investig. 2001;19(4):352-9.

51. Kismet E, Varan A, Ayabakan C, Alehan D, Portakal O, Buyukpamukcu M. Serum troponin T levels and echocardiographic evaluation in children treated with doxorubicin. Pediatr Blood Cancer. 2004;42(3):220-4. doi:10.1002/pbc.10368.

52. Gimeno E, Gomez M, Gonzalez JR, Comin J, Alvarez-Larran A, Sanchez-Gonzalez B, et al. NT-proBNP: a cardiac biomarker to assess prognosis in non-Hodgkin lymphoma. Leuk Res. 2011;35(6):715-20. doi:10.1016/j.leukres.2011.01.018.

53. Sandri MT, Salvatici M, Cardinale D, Zorzino L, Passerini R, Lentati $\mathrm{P}$, et al. N-terminal pro-B-type natriuretic peptide after high-dose chemotherapy: a marker predictive of cardiac dysfunction? Clin Chem. 2005;51(8):1405-10. doi:10.1373/clinchem. 2005.050153.

54. De Iuliis F, Salerno G, Taglieri L, De Biase L, Lanza R, Cardelli P, et al. Serum biomarkers evaluation to predict chemotherapyinduced cardiotoxicity in breast cancer patients. Tumour Biol. 2016:37(3):3379-87. doi:10.1007/s13277-015-4183-7.

55. Roziakova L, Bojtarova E, Mistrik M, Dubrava J, Gergel J, Lenkova N, et al. Serial measurements of cardiac biomarkers in patients after allogeneic hematopoietic stem cell transplantation. J Exp Clin Cancer Res. 2012;31:13. doi:10.1186/1756-9966-31-13.
56. Skovgaard D, Hasbak P, Kjaer A. BNP predicts chemotherapyrelated cardiotoxicity and death: comparison with gated equilibrium radionuclide ventriculography. PLoS One. 2014;9(5):e96736. doi: 10.1371/journal.pone.0096736.

57. Cardinale D, Sandri MT. Role of biomarkers in chemotherapyinduced cardiotoxicity. Prog Cardiovasc Dis. 2010;53(2):121-9. doi:10.1016/j.pcad.2010.04.002.

58. Witteles RM. Biomarkers as predictors of cardiac toxicity from targeted cancer therapies. J Card Fail. 2016;22(6):459-64. doi:10. 1016/j.cardfail.2016.03.016.

59. Yu AF, Ky B. Roadmap for biomarkers of cancer therapy cardiotoxicity. Heart. 2016;102(6):425-30. doi:10.1136/heartjnl2015-307894.

60. Henri C, Heinonen T, Tardif JC. The role of biomarkers in decreasing risk of cardiac toxicity after cancer therapy. Biomark Cancer. 2016;8(2):39-45. doi:10.4137/BIC.S31798.

61. Putt M, Hahn VS, Januzzi JL, Sawaya H, Sebag IA, Plana JC, et al. Longitudinal changes in multiple biomarkers are associated with cardiotoxicity in breast cancer patients treated with doxorubicin, taxanes, and trastuzumab. Clin Chem. 2015;61(9):1164-72. doi: 10.1373/clinchem.2015.241232.

62. Ky B, Putt M, Sawaya H, French B, Januzzi JL Jr, Sebag IA, et al. Early increases in multiple biomarkers predict subsequent cardiotoxicity in patients with breast cancer treated with doxorubicin, taxanes, and trastuzumab. J Am Coll Cardiol. 2014;63(8):80916. doi:10.1016/j.jacc.2013.10.061.

63. Wood PW, Choy JB, Nanda NC, Becher H. Left ventricular ejection fraction and volumes: it depends on the imaging method. Echocardiography. 2014;31(1):87-100. doi:10.1111/echo.12331.

64. Bellenger NG, Davies LC, Francis JM, Coats AJ, Pennell DJ. Reduction in sample size for studies of remodeling in heart failure by the use of cardiovascular magnetic resonance. J Cardiovasc Magn Reson. 2000;2(4):271-8.

65. Romond EH, Jeong JH, Rastogi P, Swain SM, Geyer CE Jr, Ewer MS, et al. Seven-year follow-up assessment of cardiac function in NSABP B-31, a randomized trial comparing doxorubicin and cyclophosphamide followed by paclitaxel (ACP) with ACP plus trastuzumab as adjuvant therapy for patients with node-positive, human epidermal growth factor receptor 2-positive breast cancer. J Clinical Oncol. 2012;30(31):3792-9. doi:10.1200/jco.2011.40. 0010 .

66. de Azambuja E, Procter MJ, van Veldhuisen DJ, Agbor-Tarh D, Metzger-Filho O, Steinseifer J, et al. Trastuzumab-associated cardiac events at 8 years of median follow-up in the Herceptin Adjuvant trial (BIG 1-01). J Clinical Oncol. 2014;32(20):2159-65. doi:10. 1200/jco.2013.53.9288.

67.• Zamorano JL, Lancellotti P, Rodriguez Munoz D, Aboyans V, Asteggiano R, Galderisi M, et al. 2016 ESC position paper on cancer treatments and cardiovascular toxicity developed under the auspices of the ESC committee for practice guidelines: the task force for cancer treatments and cardiovascular toxicity of the European Society of Cardiology (ESC). European Heart J. 2016;37(36):2768-801. doi:10.1093/eurheartj/ehw211. Position Paper on cancer treatments and cardiovascular toxicity from the European Society of Cardiology.

68. Cardinale D, Biasillo G, Cipolla CM. Curing cancer, saving the heart: a challenge that Cardioncology should not miss. Curr Cardiol Rep. 2016;18(6):51. doi:10.1007/s11886-016-0731-z.

69. Jerusalem G, Moonen M, Freres P, Lancellotti P. The European Association of Cardiovascular Imaging/Heart Failure Association Cardiac Oncology Toxicity Registry: long-term benefits for breast cancer treatment. Future Oncol. 2015;11(20):2791-4. doi:10.2217/ fon.15.227. 